\title{
Assessment of Pulmonary Artery Diameters in Pediatric COVID-19 Patients
}

\author{
Serdar Fidan ${ }^{1}$, Muhammed Alpaslan², Erdal Ünlü², Ramazan Dulkadir , and Ali Güneş ${ }^{3}$ \\ ${ }^{1}$ Ahi Evran University Faculty of Medicine \\ ${ }^{2}$ Ahi Evran Üniversitesi Tip Fakültesi \\ ${ }^{3}$ Ahi Evran Universitesi Tip Fakultesi
}

February 28, 2022

\begin{abstract}
ABSTRACT Background: This study was conducted to investigate the relationship between clinical course and pulmonary artery (PA) diameters in children diagnosed with COVID-19. Method: The study included 62 patients who presented COVID19 symptoms between March 2020 and April 2021. Group 1 consisted of 32 pediatric patients who were COVID-19 PCR (+), while Group 2 consisted of 30 pediatric patients who were COVID-19 PCR(-). The data were collected retrospectively from medical records. Patients who developed pneumonia due to causes other than COVID-19 and those who had a history of pulmonary hypertension or pulmonary thromboembolism were excluded. The patients were examined based on their Computerized Tomographic (CT) findings, simultaneous whole blood parameters and biochemical parameters. Results: The thoracic CT findings of 18 of the patients in Group 1 were found normal. The CT images of 14 patients showed pulmonary involvement. Among the patients with pulmonary involvement, 8 had moderate pneumonia characterized by a ground-glass pattern, and 6 had severe pneumonia indicated by consolidation and linear opacities. The right pulmonary artery, left pulmonary artery and inferior vena cava (IVC) diameters of the patients in Group 1 were significantly higher than those of the patients in Group 2 . Conclusion: The results of this study suggested that increased PA diameters in children diagnosed with COVID-19 may be accompanied by increased inflammation, high vascular resistance, hypoxemia and thromboembolic events. While it is thought that increased PA and IVC diameters are a factor that may indicate clinical deterioration in COVID-19 patients, more comprehensive studies are needed.
\end{abstract}

\section{INTRODUCTION}

COVID-19, which emerged first in China and has caused a global pandemic, causes milder clinical symptoms in children than in adults, while very few cases result in mortality ${ }^{1,2,3}$. The clinical picture of COVID-19 varies in a broad range from asymptomatic disease to multisystem dysfunction. The most frequently seen symptoms are fever, cough, nasal symptoms, diarrhea, nausea/vomiting, fatigue and respiratory distress ${ }^{3,4}$. The standard diagnostic tool for COVID-19 is the real-time polymerase chain reaction (RT-PCR) method $^{5}$. Nevertheless, studies have reported that false-negative PCR results may be encountered due to various causes $^{6,7}$. In particular, in patients who have clinical and epidemiological characteristics compatible with COVID-19 and negative RT-PCR test results, CT is also widely utilized for the early diagnosis of COVID-19 8,9 .

Although prognostic factors in COVID-19 patients have not been sufficiently explained, the relationship between lung parenchyma involvement and poor prognosis is known well ${ }^{10}$. It was previously shown that pulmonary artery (PA) expansion in $\mathrm{CT}$ scans was associated with pulmonary hypertension $(\mathrm{PH})^{11}$. $\mathrm{PH}$, which develops as a result of inflammation forming in the lungs, hypoxia and increased pulmonary vascular resistance (PVR), causes right ventricular (RV) deficiency, as well as early mortality in the case of unresponsiveness to treatment ${ }^{12}$. Therefore, changes occurring in the diameter of PA may provide new data in 
shedding light on COVID-19-related morbidity and mortality. While there are a limited number of studies investigating the relationship between PA diameters and COVID-19 in adults, the literature review that was conducted in our study did not reveal any study that examined this issue in children. In this study, it was aimed to investigate the relationship between COVID-19 and PA diameters in pediatric patients.

\section{MATERIAL AND METHOD}

This study was planned with a retrospective design. The sample included 62 pediatric patients who presented to the Pandemic Emergency Outpatient Clinic of Kırşehir Research and Training Hospital with symptoms such as fever, cough and respiratory distress between March 2020 and April 2021. The patients who were included were divided into two groups. While 32 pediatric patients who had COVID-19 PCR positivity and simultaneous thoracic CT images constituted Group 1, 30 children who had negative COVID-19 PCR results and available simultaneous thoracic CT images constituted Group 2. Patients who had pneumonia with causes other than COVID-19 and those who had a history of primary PH or pulmonary thromboembolism (PTE) were excluded. Basic demographic and clinical information was obtained from the hospital database. The test results of simultaneously collected blood samples (hemogram and biochemistry) were analyzed. The research protocol was approved by the Clinical Studies Ethics Committee of the Faculty of Medicine at Kırşehir Ahi Evran University on 07/09/2021 with the decision numbered 2021-14/151. The study was conducted in compliance with the principles of the Declaration of Helsinki.

\section{CT imaging and examination}

Thoracic images were taken with a 16-row CT device (Toshiba Alexion 16), at a slice thickness of 3mm, in the supine position and with the appropriate thoracic imaging protocol (kV:100-120,mAs:50-100). According to the CT findings, COVID-19 pneumonia was defined under three stages. At Stage 1 of the disease, the lung parenchyma was normal or close to normal. At Stage 2 (moderate pneumonia), there were ground-glass opacities that typically showed peripheral and subpleural distributions. In the presence of severe pneumonia, consolidation, linear opacities and reticular patterns were considered Stage $3^{10}$. In the study, in addition to these parameters, the right pulmonary artery diameter (RPAD), the left pulmonary artery diameter (LPAD), the main pulmonary artery diameter (MPAD), the diameter of the ascending aorta (AA) and the diameter of the inferior vena cava (IVC) were also measured (Fig.1,Fig.2). Each CT scan was shown in a computer interface where the researcher identified the axial part of CT showing the splitting of the main pulmonary artery and measured arterial diameters manually.

\section{STATISTICAL ANALYSIS}

The data were analyzed using the SPSS (Statistical Package for the Social Sciences) 18.0 software. The measured variables are presented with mean \pm standard deviation values, while the categorical variables are presented with frequency and percentage values. Kolmogorov-Smirnov or Shapiro-Wilk tests were used to assess the distribution of the data. The numerical values that were compatible with normal distribution were compared using t-test or one-way analysis of variance (ANOVA). Those that did not show normal distribution were compared using Mann-Whitney U test or Kruskal-Wallis test. Pearson's chi-squared test was used to analyze the categorical variables. $\mathrm{p}<0.05$ was accepted as statistically significant.

\section{RESULTS}

The mean age of the patients in Group 1 was $12.27 \pm 4.835$ years, while $15(46.8 \%)$ were male, and $17(54.2 \%)$ were female. The mean age of the patients in Group 2 was $12.22 \pm 4.818$ years, while 11 (36.6\%) were male, and $19(63.4 \%)$ were female. There was no significant difference between the two groups in terms of their age and sex distributions $(\mathrm{p}>0.05)$. None of the patients in Groups 1 and 2 had a history of comorbidity or medication use.The CT findings of 18 (56.25\%) of the patients in Group 1 were assessed to be normal. Noticeable pulmonary involvement was present in 14 patients. Among the patients who had pulmonary involvement, $8(25 \%)$ had moderate pneumonia characterized by ground-glass patterns, while $6(18.75 \%)$ had severe pneumonia indicated by consolidation and linear opacities. The right pulmonary artery, left pulmonary artery and inferior vena cava diameters of Group 1 were significantly larger than those in Group 
2 (respectively, $\mathrm{p}=0.031, \mathrm{p}=0.008$ and $\mathrm{p}=0.013$ ), whereas the differences between the mainpulmonaryartery and ascending aorta diameters of the two groups were not significant $(\mathrm{p}>0.05)$ (Table-1).In the examination of the whole blood parameters of the groups, the leukocyte counts (WBC) of Group 2 were significantly higher than those of Group $1(\mathrm{p}=0.002)$, while there was no significant difference in the other parameters $(\mathrm{p}>0.05)$ (Table-2). In the comparison of the biochemical parameters of the groups, while the ALT value in Group 1 was significantly higher $(\mathrm{p}<0.05)$, the other parameters did not differ significantly between the two groups ( $\mathrm{p}>0.05)$ (Table-3).

\section{DISCUSSION}

Thoracic CT is prevalently used to determine the diagnosis, follow-up and prognosis of COVID-19 pneumonia. As the stage of pneumonia in COVID-19 increases, disease-related mortality increases ${ }^{10,13}$. Different studies have reported varying rates of pulmonary involvement ${ }^{14}$. In this study, while the thoracic CT findings of 18 patients in Group 1 were normal, 8 patients had Stage 2 pneumonia, and 6 had Stage 3 pneumonia. None of the patients in this study who were diagnosed with COVID-19 had a comorbid condition, and no mortality occurred.

In addition to parameters that explain the characteristics and severity of lung parenchyma involvement, vascular measurements can also be analyzed on thoracic CT images. Enlarged PA diameters on CT images were studied as a method of screening and diagnosing $\mathrm{PH}^{15}$. $\mathrm{PH}$ can develop in relation to many causes such as idiopathic, genetic, cardiac, pulmonary, vascular and medication-related causes ${ }^{16}$. CT is suitable for evaluating interstitial lung disease, vascular anomalies and thromboembolic factors, which are among the secondary causes of $\mathrm{PH}^{17}$. In this study, it was found that the PA diameters of the pediatric children who had COVID-19 infection on their thoracic CT images were larger. A single CT examination in pediatric COVID-19 patients might not provide clear information about the etiology of PA expansion. However, the demographic characteristics of the patients and the absence of comorbid diseases suggested that the cause of the PA expansion was an acute complication developing secondarily to the COVID-19 infection. Still, COVID-19 pneumonia can present in the form of hypoxemia as a result of ventilation-perfusion mismatch, increased PVR and expansion in the diameter of PA.

The morphology of IVC is frequently used to estimate right atrial pressure; however,the relationship between IVC morphology and RV outcomes has not been clarified completely ${ }^{18}$. Dilated IVC cases were found to be associated with RV dysfunction, high central venous pressure, and high pulmonary artery pressure ${ }^{19}$. High PVR can lead to IVC dilatation along with PH and RV deficiency. Some studies showed that IVC dilatation was one of the early indicators of disrupted hemodynamics in shock patients ${ }^{19}$. The mechanism of RV dilatation is multifactorial, and it involves thrombotic events, hypoxemic vasoconstriction, cytokines and direct viral damage. In COVID-19 patients, RV dilatation was determined to be associated with a poor prognosis ${ }^{20}$. In this study, the diameter of IVC was significantly larger in the COVID-19 patients, but no significant finding was detected about RV dilatation.

In pediatric patients, the laboratory findings of COVID-19 are non-specific. Leukocyte and platelet counts may be normal, increased, or decreased ${ }^{4,21}$. Severe infection is frequently accompanied by lymphopenia ${ }^{22}$. In this study, in Group 1, 12 patients $(40 \%)$ had leukopenia, and $3(9.3 \%)$ had lymphopenia. While the leukocyte counts in Group 2 were significantly higher, there was no significant difference between Groups 1 and 2 in terms of the other whole blood parameters. Likewise, in most COVID-19 patients, CRP, ferritin, fibrinogen, D-dimer, LDH, CPK, ALT and AST levels may be found higher in proportion to the severity of the infection ${ }^{4}$. There was no significant difference between the two groups in this study regarding their CRP, ferritin, D-dimer, fibrinogen, LDH, CPK or AST values among biochemical parameters. The finding that clinical symptoms of COVID-19 emerged in Group 1 without a noticeable increase in acute phase reactants may be an indicator that different immune and inflammatory mechanisms are in place in children. Although these findings could partly explain the milder course of COVID-19 in children than in adults, they suggested that the mechanism is more complex. Liver damage can be seen in COVID-19 infections. The mechanisms of this damage may include direct cholangiocyte and hepatocyte damage or accompanying conditions such as hypoxia, sepsis and multiorgan dysfunction caused by antiviral medication use, systemic 
inflammatory response and respiratory distress syndrome ${ }^{23}$. Temporary disruptions in liver function tests may be encountered in COVID-19 infection cases, and these might not affect liver-related morbidity and mortality $^{23}$. In this study, the ALT levels of Group 1 were significantly higher than those of Group 2. Anomalies in liver function tests are usually encountered alongside increased enzyme activities in muscles and the heart. In COVID-19 cases, myocardial injury defined by increased troponin levels occurs especially due to hypoxia, sepsis, systemic inflammation, pulmonary thrombosis and embolism, in addition to severe respiratory airway infection and non-ischemic myocardial processes like cardiac adrenergic hyperstimulation during a cytokine storm ${ }^{24}$. In this study, the troponin I levels were higher in Group 1 than Group 2, but the difference was not statistically significant.There was no significant difference between the groups regarding their D-dimer levels. Many studies have demonstrated that increased D-dimer levels in adult COVID-19 patients are an indicator of PTE ${ }^{25,26}$. COVID-19-related PTE cases in the pediatric population are very rare $^{27}$. This suggests that there was no increased risk of PTE in the patients in Group 1, and their high troponin I levels could be associated with hypoxia and systemic inflammatory response.

Consequently, the results of this study indicated that increased diameters of PA and IVC as a result of increased inflammation, high vascular resistance and hypoxemia in pediatric COVID-19 patients may be an early warning sign related to potential cardiopulmonary complications. To propose an exact explanation on this topic, there is a need for more comprehensive studies supported by larger datasets.

\section{LIMITATIONS}

The retrospective nature and small sample size of the study were its most significant limitations. The measurement of the arterial diameters of the patients with a single CT scan at the time of their presentation to the hospital and the lack of CT data before and after their COVID-19-related examination were also among the limitations of this study. Moreover, another limitation was that this study did not include electrocardiographic and echocardiographic tests that would be required to assess the cardiac condition of the patient better.

\section{References}

1. World Health Organization . Coronavirus disease 2019 (COVID-19): Situation report - 64. 2020.

2. Lu X, Zhang L, Du H, Zhang J,Y Li Y, Qu J, Zhang V, Wang Y, Bao S, Li Y, et al. SARS-CoV-2 infection in children. N Engl J Med. 2020;382:1663-1665.

3. Ludvigsson JF. Systematic review of COVID-19 in children shows milder cases and a better prognosis than adults. Acta Paediatr. 2020 Jun;109(6):1088-1095.

4. de Souza TH, Nadal JA, Nogueira RJN, Pereira RM, Brandao MB. Clinical manifestations of children with COVID-19: A systematic review. Pediatr Pulmonol. 2020 Aug;55(8):1892-1899.

5. Tang YW, Schmitz JE, Persing DH, Stratton CW. Laboratory Diagnosis of COVID-19: Current Issues and Challenges. J Clin Microbiol. 2020 May 26;58(6).

6. Shi F, Wang J, Shi J, Wu Z, Wang Q, Tang Z, He K, Shi Y, Shen D. Review of artificial intelligence techniques in imaging data acquisition, segmentation, and diagnosis for COVID-19. IEEE Rev Biomed Eng. 2021;14:4-15.

7. Hernandez-Huerta MT, Mayoral LPT, Navarro LMS, Mayoral-Andrade G, Perez-Campos Mayoral E, Zenteno E,Campos EP. Should RT-PCR be considered a gold standard in the diagnosis of COVID-19. J Med Virol. 2020;92(11):2312-3.

8. Huang P, Liu T, Huang L, Liu H, Lei M, Xu W, Hu X, Chen J, Liu B. Use of chest CT in combination with negative RT-PCR assay for the 2019 novel coronavirus but high clinical suspicion. Radiology 2020 Apr;295(1):22-23. doi: 10.1148/radiol.2020200330.

9. Fang Y, Zhang H, Xie J, Lin M, Ying L, Pang P, Ji W. Sensitivity of chest CT for COVID-19: comparison to RT-PCR. Radiology. 2020;296(2):E115-117.

10. Li Y, Yang Z, Ai T, Wu S, Xia L. Association of "initial CT" findings with mortality in older patients with coronavirus disease 2019 (COVID-19). Eur Radiol. 2020 Nov; 30(11):6186-6193.

11. Truong QA, Bhatia HS, Szymonifka J, Zhou Q, Lavender Z, Waxman AB, Semigran MJ, Malhotra R. A four-tier classification system of pulmonary artery metrics on computed tomography for the 
diagnosis and prognosis of pulmonary hypertension. J Cardiovasc Comput Tomogr. 2018;12:60-66. doi: $10.1016 /$ j.jcct.2017.12.001.

12. Tonelli AR, Arelli V, Minai OA, Newman J, Bair N, Heresi GA, Dweik RA. Causes and circumstances of death in pulmonary arterial hypertension. Am J Respir Crit Care Med 2013;188:365-369.

13. Liu F, Zhang Q, Huang C, Shi C, Wang L, Shi N, Fang C, Shan F, Mei X, Shi J, et al. (2020) CT quantification of pneumonia lesions in early days predicts progression to severe illness in a cohort of COVID-19 patients. Theranostics 10:5613-5622.

14. Cui X, Zhao Z, Zhang T, Guo W, Guo W, Zheng J, Zhang J, Dong C, Na R, Zheng L, et al. Children with coronavirus disease 2019: A review of demographic, clinical, laboratory, and imaging features in pediatric patients. J Med Virol. 2021 Feb;93(2):1057-1069. doi: 10.1002/jmv.26398. Epub 2020 Sep 28.

15. Chaudry G, MacDonald C, Adatia I, Gundogan M, Manson D. (2007) CT of the chest in the evaluation of idiopathic pulmonary arterial hypertension in children. Pediatr Radiol 37:345-350.

16. Hansmann G.Pulmonary Hypertension in Infants, Children, and Young Adults. J Am Coll Cardiol. 2017 May 23;69(20):2551-2569. doi:10.1016/j.jacc.2017.03.575.

17. Galie N, Humbert M,Vachiery JL, Gibbs S, Lang I, Torbicki A, Simonneau G, Peacock A, Noordegraaf AV, Beghetti M, et al. 2015 ESC/ERS Guidelines for the diagnosis and treatment of pulmonary hypertension: The Joint Task Force for the Diagnosis and Treatment of Pulmonary Hypertension of the European Society of Cardiology (ESC) and the European Respiratory Society (ERS) Endorsed by: Association for European Paediatric and Congenital Cardiology (AEPC), International Society for Heart and Lung Transplantation (ISHLT). Eur Heart J 2016;37:67-119. doi: 10.1093/eurheartj/ehv317.

18. Nath J, Vacek JL, Heidenreich PA. A dilated inferior vena cava is a marker of poor survival. Am Heart J 2006; 151(3):730-735. doi.org/10.1016/j.ahj.2005.04.023.

19. Garcia-Montilla R, Mukundan S, Heitner SB, Khan A. Inferior vena cava dilation predicts global cardiac dysfunction in acute respiratory distress syndrome: A strain echocardiographic study.Echocardiography.2021 Feb;38(2):238-248. doi: 10.1111/echo.14970.

20. Argulian E, Sud K, Vogel B, Bohra C, Garg VP, Talebi S, Lerakis S, Narula J. Right ventricular dilation in hospitalized patients with COVID-19 infection. JACC Cardiovasc Imaging. 2020;13(11):2459-2461. doi: 10.1016/j.jcmg.2020.05.010.

21. Xia W, Shao J, Guo Y, Peng X, Li Z, Hu D. Clinical and CT features in pediatric patients with COVID-19 infection: Different points from adults. Pediatr Pulmonol. 2020 May;55(5):1169-1174. doi: $10.1002 /$ ppul.24718.

22. Tan L, Wang Q, Zhang D, Ding J, Huang Q, Tang YQ, Wang Q, Miao H. Lymphopenia predicts disease severity of COVID-19: a descriptive and predictive study. Signal Transduct Target Ther. 2020;5(1):33. doi: 10.1038/s41392-020-0148-4.

23. Portincasa P, Krawczyk M, Machill A, Lammert F, Di Ciaula A.Hepatic consequences of COVID-19 infection. Lapping or biting? Eur J Intern Med. 2020 Jul;77:18-24. doi: 10.1016/j.ejim.2020.05.035.

24. Imazio M, Klingel K, Kindermann I, Brucato A, De Rosa FG, Adler Y, De Ferrari GM. COVID-19 pandemic and troponin: indirect myocardial injury, myocardial inflammation or myocarditis? Heart. 2020 Aug;106(15):1127-1131. doi: 10.1136/heartjnl-2020-317186.

25. Garcia-Oliv'e I, Sintes H, Radua J, Abad Capa J, Rosell A. D-dimer in patients infected with COVID-19 and suspected pulmonary embolism, Respir. Med. 169 (2020), 106023, doi: 10.1016/j.rmed.2020.106023.

26. Fauvel C, Weizman O, Trimaille A, Mika D, Pommier T, Pace N, Douair A, Barbin E, Fraix A, Bouchot O. Pulmonary embolism in COVID-19 patients: a French multicentre cohort study, Eur. Heart J. 41 (32) (2020)3058-3068, doi: 10.1093/eurheartj/ehaa500.

27. Chima M, Williams D, Thomas NJ, Krawiec C. COVID-19-Associated Pulmonary Embolism in Pediatric Patients. Hosp Pediatr. 2021 Jun;11(6):90-94. doi: 10.1542/hpeds.2021-005866.

\section{Hosted file}

covid-19 -Pediatric pulmonology Table.docx available at https://authorea.com/users/462832/ 
articles/558136-assessment-of-pulmonary-artery-diameters-in-pediatric-covid-19-patients
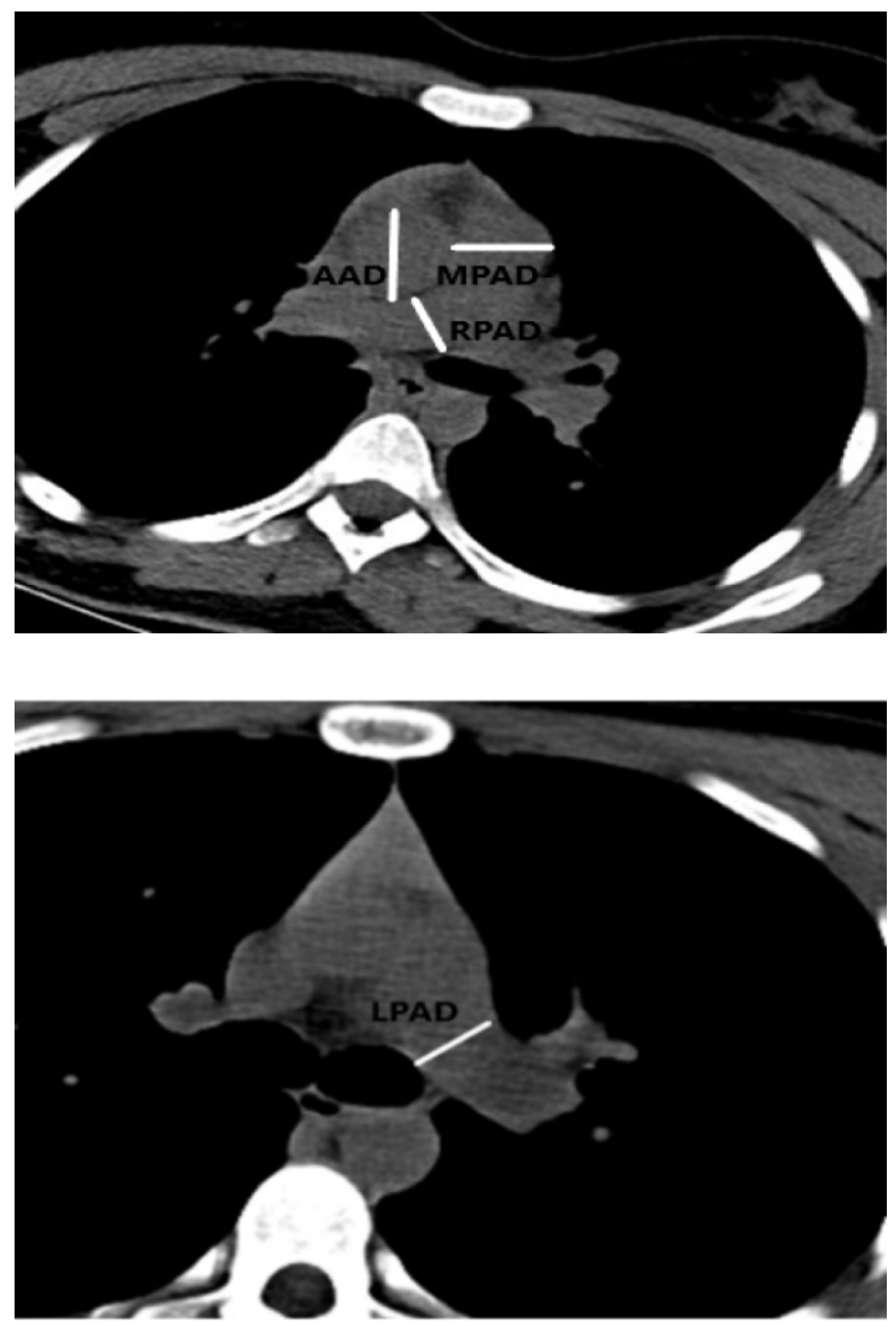